\title{
Digital computer estimation of eye fixations
}

\author{
RICHARD L. MASON \\ Naval Underwater Systems Center \\ New London Laboratory, New London, Connecticut 06920
}

\begin{abstract}
A method is presented for estimating eye fixations to the degree of accuracy desired. In the process, the quantity of data is greatly reduced so that analysis can be accomplished at the end of each experimental run. The algorithm is particularly suitable for "slow" computers because the analog-to-digital conversion rate can be low and because the data reduction process decreases the number of calculations required to obtain the analytical results.
\end{abstract}

Studies of eye fixations provide large amounts of data with accompanying problems of analysis and storage. For processing such eye-movement data, this paper presents an on-line method in which the raw data are reduced to numbers representing only the location and duration of each fixation. The reduced quantity and form of the processed data permit analysis of the eye fixations following each experimental run.

The problems associated with measuring eye movements were considered in the On-line Eye-Movement Recording Systems symposium at the national conference last year (1974) and are not addressed here.

\section{ASSUMPTIONS}

The following assumptions are made for the digital processor described, although Assumptions 1 and 2 can be altered to suit the experimental conditions and the final data analysis to be done:

(1) The fixation point is located by means of an angular measurement. Most methods of measuring fixations involve measuring the angle of the eye, with the subject's head fixed and the display at a known distance. The actual fixation point on the display is then calculated from the known angle and distance.

(2) Only the duration and location of the fixation are desired. Saccadic parameters are not considered, and microsaccades occurring around the fixation point are treated as noise.

(3) A minimum accuracy for the location of the fixations can be determined by the experimenter. This accuracy is represented by $\pm \Delta$, and in the examples given below $\Delta=1 \mathrm{deg}$.

\section{ESTIMATION OF THE FIXATION POINT}

Figure 1 shows hypothetical signals for the vertical and horizontal components of a fixation. Each dotted line represents a fixation, and the variation about the fixation can be considered to be noise. The best estimate of the fixation point is the average of the signal during the fixation, i.e., between movements. Since there are many small movements, one must be able to determine when a change in fixation point has occurred in order to know when to start a new estimate (average).

This problem is circumvented by arbitrarily assuming that a change in fixation point has occurred when the signal deviates from the average by more than some value $\Delta$. Thus a movement is defined by $\Delta$, and all the digital samples between moves are averaged to form the estimate of the fixation.

The value chosen for $\Delta$ determines the minimum accuracy of the fixation estimate because all moves less than $\Delta$ are averaged out. This decision-how small a move about the fixation point can be ignored-must always be made when analyzing eye fixation data. It is used in this program for an immediate reduction in the quantity of data.

\section{THE ALGORITHM}

Figure 2 is a block diagram of the basic algorithm for estimating the fixations. The horizontal $(x)$ and vertical (y) components of eye fixations are considered separately, but the algorithm could be modified to combine these components. Separation, however, makes the pro-

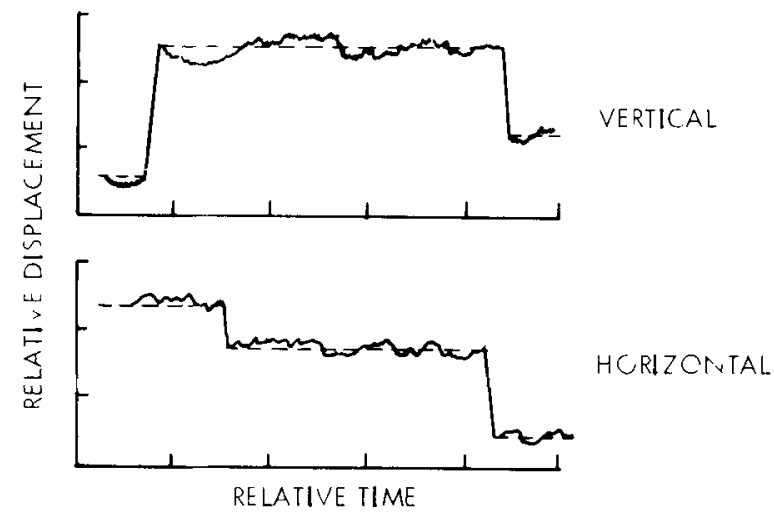

Figure 1. Components of a fixation with noise (hypothetical case). The solid lines represent the observed signals and the dashed lines represent fixations. 


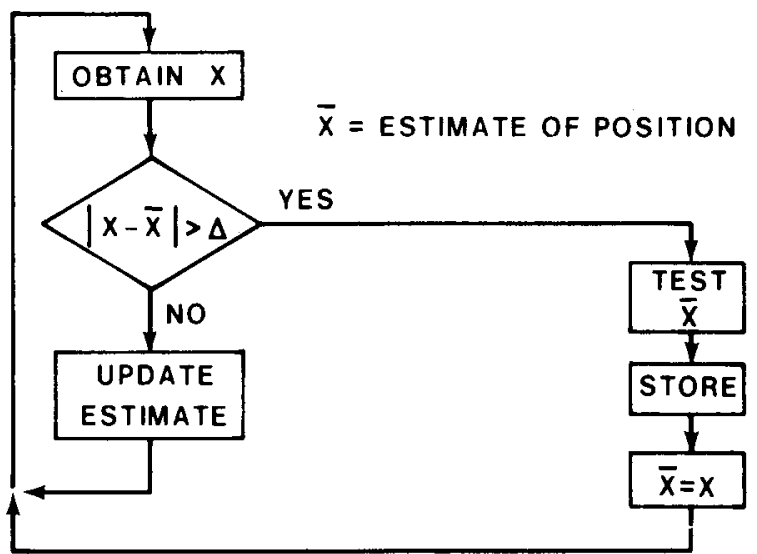

Figure 2. Block diagram of fixation data processing algorithm.

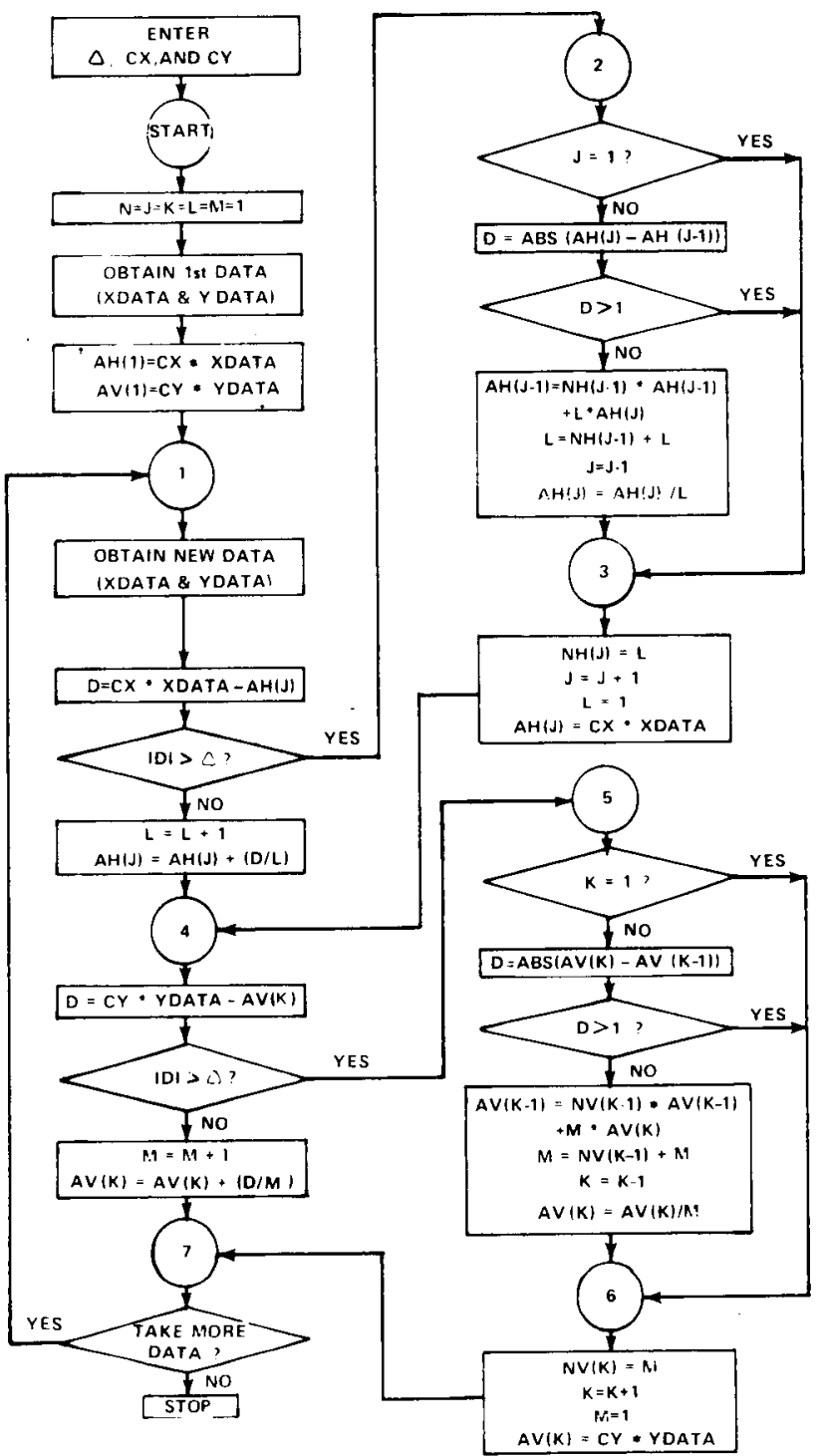

Figure 3. Detailed program flow chart for data processing algorithm [CX and CY are gains, $A H(J)$ and $A V(K)$ are the estimates of the horizontal and vertical fixations, and $N H(J)$ and NV(K) are the numbers of samples in the estimates]. gramming easier to follow, and for many experiments it either does not matter or there are few diagonal moves.

The input to the algorithm is the sequence of digital samples obtained from the analog-to-digital (A/D) converter, and the output is the location and duration of each fixation. Each input sample is compared with the current estimate of the fixation; if they differ by less than $\Delta$, the estimate is updated with that sample, and the next sample is considered. When an input $x$ deviates from the estimate by more than $\Delta$, the estimate is terminated. Then a check is made between the estimates of the current fixation and the previous fixation. If the difference between these successive estimates is less than $\Delta$, the two estimates are combined. The nexi estimate is started with $\mathrm{x}$ as its initial value.

Figure 3 is the detailed flow chart of the algorithm. This program, with $\Delta=1 \mathrm{deg}$, was used to make the estimates of fixations given below.

\section{PRACTICAL APPLICATIONS}

The examples given here are from a study of the visual search patterns of trained sonar operators viewing a rectangular sonar display (Mason, 1975). The study was an analysis of operators' eye fixations on the display while trying to detect a target (a vertical column with significantly more marks than would be expected from noise alone).

Figure 4 shows examples of measured raw data and the fixation estimates derived. The figure indicates that high-frequency noise in the input data has been eliminated in the processed data, yet the rapid changes of fixation point that are characteristic of eye movements have been retained. The reduction in the amount of data storage required is also illustrated by this figure; instead of storing each digital sample $(64 / \mathrm{sec})$, only the fixations themselves (approximately $3 / \mathrm{sec}$ ) are stored.

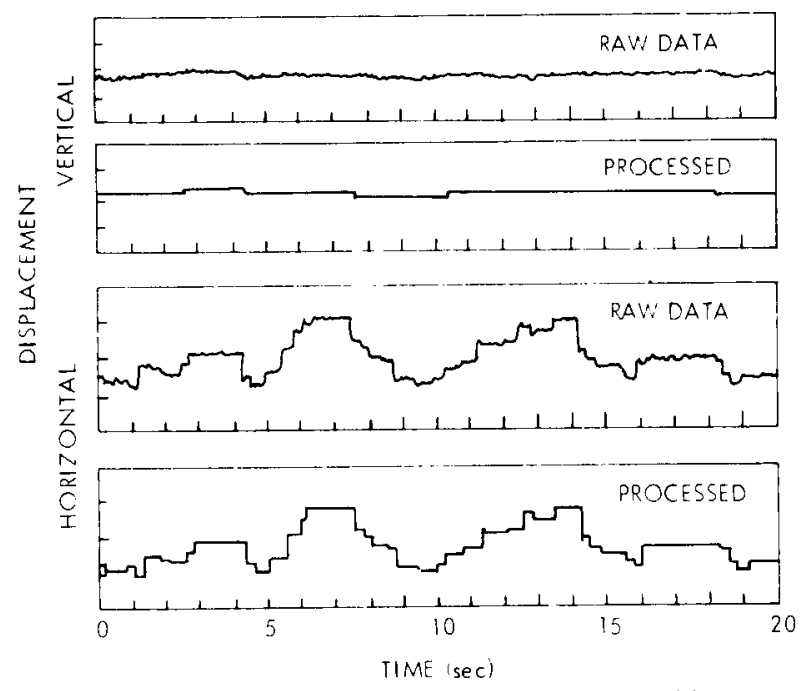

Figure 4. Input data and fixation estimates derived by means of the algorithm. 
When the program output data are combined to obtain statistics of the subjects' eye fixations, interpretations of the results should be based on the characteristics of the estimations. The following analyses of sonaroperation fixation data are typical of the type done for eye fixations, and the effect of the algorithm is considered for each type.

Figure 5 shows the relative amount of time spent in various lengths of fixation while the operators searched for a target. Figure 5b, an expanded version of Figure $5 \mathrm{a}$, shows the 0 - to .0156 -sec block that represents those estimates for which the algorithm output consists of a single digital sample $(1 / 64 \mathrm{sec})$. This block requires special interpretation.

The time in the 0 - to .0156 -sec block should be interpreted as eye-movement time. When the eye is moving between fixation points, the A/D converter produces a series of samples that differ by $1 \mathrm{deg}$ or more in magnitude, and so the algorithm gives an output that includes these single samples as estimates. Therefore, the time spent in making eye movements $(>1 \mathrm{deg})$ is represented by these single sample estimates, and that total amount of time is indicated by the 0 - to $.0156-\mathrm{sec}$ block in Figure 5b.
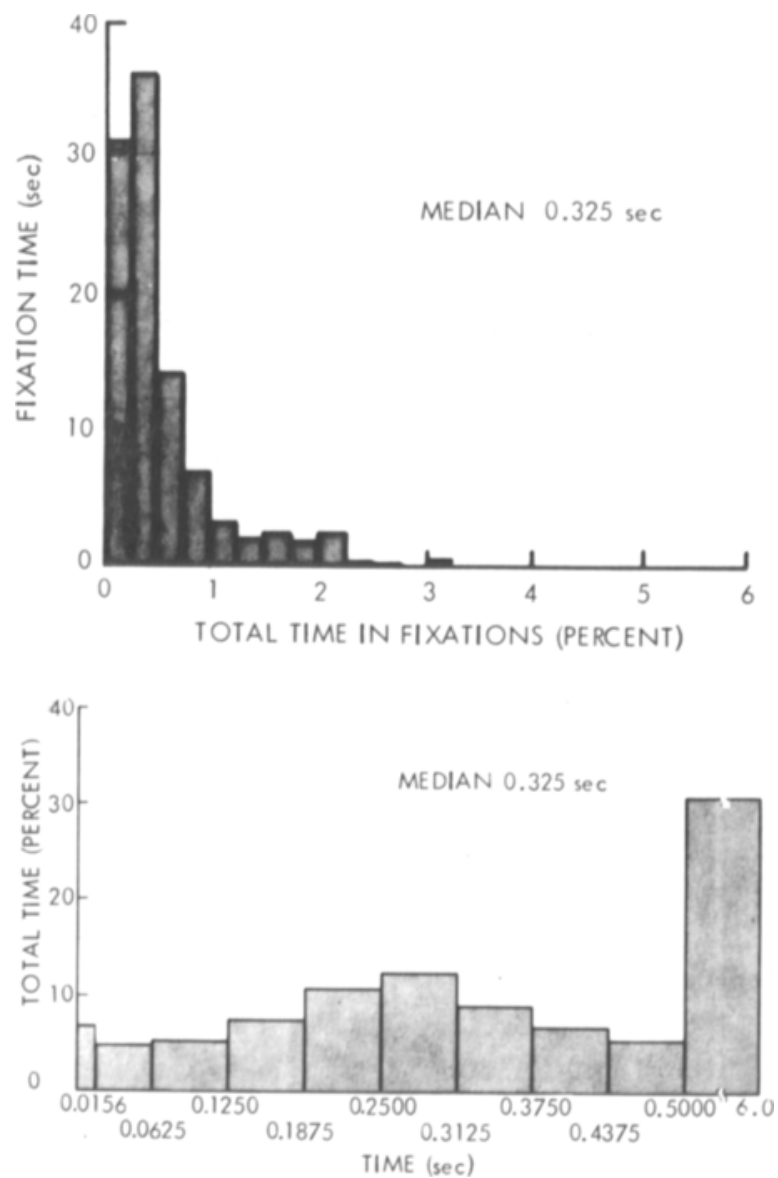

Figure 5. Distribution of fixation periods as portions of total viewing time (data for three operators combined). (a) Full scale. (b) Expanded scale, 0 to $.5 \mathrm{sec}$.

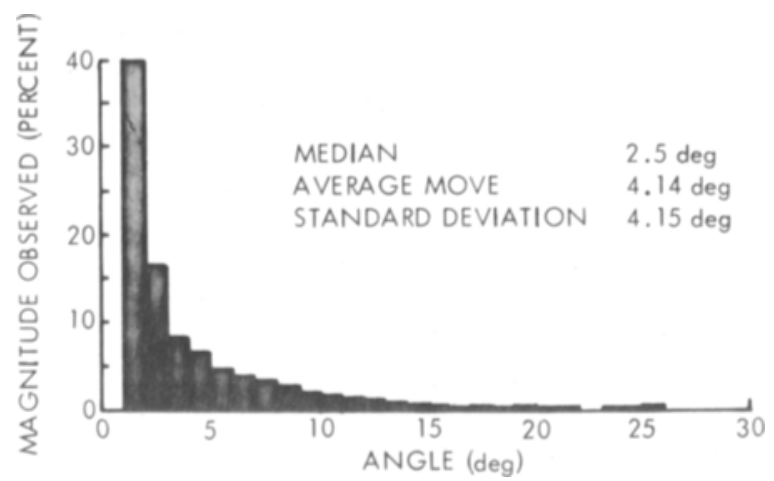

Figure 6. Distribution of eye-movement magnitudes.

The length of estimate to be interpreted as caused by eye movements depends on $\Delta$ and on the sample rate used for the $A / D$ converter. If the $A / D$ converter samples at a rate faster than the one used for the data in this example, then estimates two or three samples long should be interpreted as eye movements.

Figure 6 is a histogram of the length of eye movements made by the sonar operators. The data for this figure were also calculated from the output of the program; therefore, there are no moves shown in the 0 - to 1 -deg block of the figure (since $\Delta$ was set at $1 \mathrm{deg}$, all moves of 1 deg or less are averaged out by the program and cannot appear in the figure).

When the magnitude of the difference between fixation points is the desired parameter, then the small movements around the fixation point are not wanted, and, in fact, obscure the desired results. Therefore, the program output is particularly suitable for making the type of data analysis shown in Figure 6.

Figure 7 shows the visual coverage of a sonar operator viewing one display presentation. The circles indicate the display areas represented by $2.5 \mathrm{deg}$ of visual angle, and the center of each circle is at the point estimated by the program for each fixation.

The output of the program is particularly good for showing the visual coverage, as in Figure 7 . When the one-sample estimates are eliminated, there is no false indication of coverage as the eye moves from one fixation

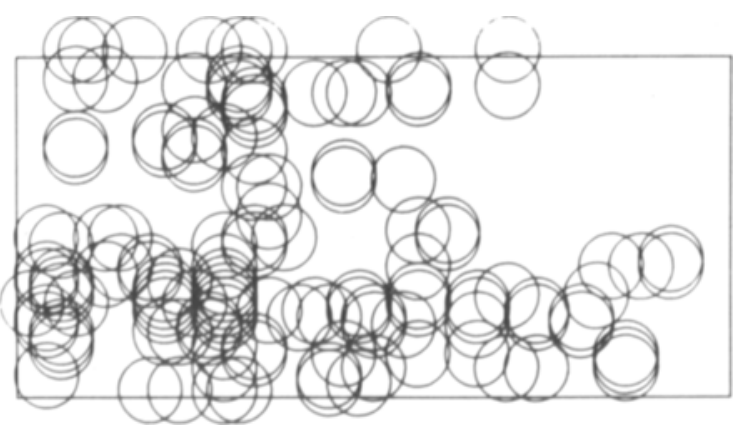

Figure 7. Visual coverage of a display presentation. Each circle represents a visual angle of $2.5 \mathrm{deg}$. 
point to the next, and the averaging done during the fixations eliminates the jitter that would be caused by small movements around the fixation point.

The algorithm given can be modified in order to study other parameters. For instance, in a study of saccades, the fixations would be of little interest, but sequential one- or two-sample estimates could be identified as saccades, and their properties could then be studied. Similarly, the microsaccades occurring during a fixation could be analyzed by considering the raw data during the fixation and their deviation from the fixation value. This type of study would be particularly applicable to the method given; the experimenter woud simply define the degree of movement to be considered a microsaccade, estimate the fixation for $\Delta$ greater than this value, and then subtract the estimates from the raw data to obtain the microsaccades.

\section{REFERENCE NOTE}

1. Mason. R. L. Visual sampling on a simulated DIMUS-type display. Technical Report 4861. New London, Conn: Naval Underwater Systems Center, 1975.

\section{REFERENCE}

Proceedings of the symposium on on-line eye-movement recording systems at the 1974 National Conference on the Use of On-Line Computers in Psychology. Behavior Research Methods \& Instrumentation, 1975, 7, 201-219. 